\title{
ATRAÇÃO DE MOSQUITOS (DIPTERA: CULICIDAE) POR LÂMPADAS INCANDESCENTES E FLUORESCENTES
}

\section{Alessandro Barghini( ${ }^{(1)}$, Paulo Roberto Urbinatti( ${ }^{(2)}$ \& Delsio Natal(2)}

\begin{abstract}
Fluorescent and incandescent light attractiveness to mosquitos (Diptera: Culicidae) - Rural electrification programs in remote areas based on both photovoltaic and traditional installations, could introduce a new anthropic factor, which is associated with Culicidae. To evaluate differences in mosquitoes attractiveness in relation to types of lamps two New Jersey traps were used: one with a $40 \mathrm{~W}$ incandescent lamp and the other with the same light intensity, a $9 \mathrm{~W}$ fluorescent bulb, compact, with an electronic reactor with $6.200 \mathrm{~K}$ of light temperature. The traps were set in two chosen areas according time and place previously established in a total of 11 collections from January and April 2002. The results show that the fluorescent lamp attracted 1,3 more culicidae than the incandescent lamp, in both areas a total of 806 specimens were collected including Aedes albopictus Skuse, 1894, Ochlerotatus scapularis (Rondani, 1848), Anopheles albitarsis Lynch Arribálzaga, 1878, Culex (Culex) nigripalpus, Theobald, 1901, among others. Studies should be carried out before the rural electrifications programs are implemented in order to evaluate the epidemiological consequences of such enterprises.
\end{abstract}

Key words: Culicidae, impacts, rural electrification

(1) Laboratório de Estudos Evolutivos Humanos, Departamento de Ecologia, Instituto de Biociências, Universidade de São Paulo, Rua do Matão 321, CEP: 05508-900 São Paulo, SP, Brasil. E-mail: barghini@terra.com.br; (2) Departamento de Epidemiologia da Faculdade de Saúde Pública, Universidade de São Paulo, Av. Dr. Arnaldo 715, CEP: 012406-904 São Paulo, SP, Brasil.

Recebido em: 12/08/2004

Aceito em: 04/11/2004. 


\section{Resumo}

Programas de eletrificação rural em áreas isoladas, implantados com base em instalações fotovoltaicas e tradicionais, introduziriam um novo fator antrópico, que levantam suspeitas de associação à transmissão de doenças por culicídeos. Para testar atratividade de mosquitos por diferentes tipos de lâmpadas, foram utilizadas duas armadilhas tipo New Jersey, uma com lâmpada incandescente de 40 watts, e a outra, provida de bulbo fluorescente, compacta e com reator eletrônico de cor de igual intensidade luminosa, de 9 watts, com luz de temperatura de $6.200 \mathrm{~K}$. Essas armadilhas operaram em duas áreas, em tempo e locais padronizados, totalizando 11 coletas, no período de janeiro a abril de 2002. Os resultados mostram que a lâmpada fluorescente atraiu 1,3 vezes mais culicídeos em relação à incandescente nas duas áreas. Foram coletados no total 806 espécimes, destacando-se: Aedes albopictus Skuse, 1894, Ochlerotatus scapularis (Rondani, 1848), Anopheles albitarsis Lynch Arribálzaga, 1878, Culex (Culex) nigripalpus Theobald, 1901, entre outros. Sugere-se que sejam realizados estudos antes da efetivação de programas de eletrificação rural para avaliar as conseqüências epidemiológicas desses empreendimentos.

Palavras-chave: Culicidae, impactos, eletrificação rural

\section{Introdução}

A atração de insetos à luz é fato bem conhecido, já citado pelo poeta grego Eurípides, e utilizado, a partir do século XVIII, pelos entomólogos nas coletas. Várias explicações foram dadas a esse fenômeno (MazokhinPorshnyakov , 1969; Dethier, 1963; Goldsmith, 1970; Clements, 1999), porém, na teoria mais corrente, a atração é atribuída a uma desordem no sistema de localização do inseto, que o induz a esbarrar ou a circular em volta de uma fonte luminosa. A atração pela luz artificial depende da sensibilidade dos receptores a diferentes comprimentos de onda. Nos insetos em geral, e nos culicídeos em particular, a sensibilidade das rodopsinas se verifica em comprimentos de ondas distintos em relação àqueles dos mamíferos e do homem. Nos culicídeos a sensibilidade à luz está entre 350 e 600 nm, no homem se encontra entre 420 e 680 nm (Goldsmith, 
1970; Brioscoe \& Chittka, 2001). Na prática o inseto percebe a radiação ultravioleta $A$, que para o homem é invisível.

Esse fato tinha um valor relativamente pequeno quando as principais fontes de iluminação eram as lâmpadas incandescentes. Com espectro contínuo próximo a irradiância de um corpo negro, elas tinham luz calibrada para a visão humana. Com a introdução das lâmpadas de tipo de descarga (fluorescentes, vapor de sódio, vapor de mercúrio, vapores metálicos), o espectro de irradiância das mesmas não é contínuo, e depende da natureza dos gases ou dos fósforos utilizados (IESNA, 1993). A temperatura da cor das lâmpadas é apenas o resultado para o olho humano de um conjunto de emissões em diferentes bandas espectrais. Ao lado das bandas de irradiância úteis para vista do homem, essas lâmpadas registram emissões nas bandas de ultravioleta A, B e C (Bergman et al., 1995; McGowan, 1989).

Diante das evidências colocadas e frente às mudanças que vêm ocorrendo na modernização do sistema de iluminação em nosso país, justificam-se as preocupações que motivaram a presente pesquisa. A iluminação artificial pode significar risco, principalmente em áreas de baixa concentração humana, pois nas localidades menores e sujeitas ao isolamento, o aumento da densidade de vetores atraídos pelos novos sistemas de iluminação, dificilmente receberá a atenção devida por parte dos serviços de controle. Como exemplo, essa realidade poderá complicar a transmissão da malária na região amazônica.

Este trabalho tem como objetivo verificar a atração de culicídeos em teste de campo, utilizando-se fontes luminosas de irradiância em comprimentos de onda diferentes, regularmente utilizadas nos sistemas de iluminação, com base na eficiência relativa de capturas. A interpretação dos resultados poderá ressaltar a importância de serem conduzidos estudos de avaliação das respostas que os mosquitos podem apresentar, frente à eletrificação rural e à expansão de núcleos urbanos em nosso país.

\section{Material e Métodos}

Antes do início das capturas dos mosquitos, foram realizadas as medições das lâmpadas entre 290 e 900 nm no laboratório no Instituto de Pesquisas Tecnológicas, utilizando os seguintes equipamentos: Radiômetro digital, marca Optronic, Modelo 730 A, monocromador Optronic $740 \mathrm{~A}$ e controlador de varredura automática de comprimento de onda do 
monocromador marca Optronic modelo 740-1C, utilizando o procedimento interno IPT DME-LO-PE-020. A medição radiométrica fornece apenas a irradiância espectral em todos os comprimentos de onda medidos. Considerada a diferente sensibilidade das rodopsinas dos insetos e do homem, a avaliação da intensidade da luz para as diferentes espécies deve ser realizada integrando a irradiância espectral na curva própria da sensibilidade das rodopsinas. Para avaliar a igual intensidade da irradiância sobre o homem, foi utilizado Luxîmetro Grossen, modelo Electronic 2, o qual possui circuito integrador da luz, conforme a curva $\lambda$ prescrita pela CIE.

Para a execução do teste foram utilizadas duas armadilhas luminosas do tipo New Jersey (Service, 1993; Reinert, 1989), uma com lâmpada comum incandescente de 40 watts e 127 volts; a outra, fluorescente de 9 watts, de luz "branca", temperatura de cor $6.200 \mathrm{~K}$ e com reator eletrônico de $20.000 \mathrm{~Hz}$, para evitar os flickers (batimentos da luz), presentes nas lâmpadas com reator eletromagnético de $60 \mathrm{~Hz}$, aos quais os insetos são sensíveis. A emissão de luz era equivalente nas duas lâmpadas utilizadas.

As armadilhas foram alocadas em pontos fixos, a uma altura de $250 \mathrm{~cm}$, conforme o padrão de captura das armadilhas New Jersey (Reinert, 1989), simulando as condições de uma iluminação externa domiciliar. A cada coleta as lâmpadas eram trocadas para evitar que a capacidade de aspiração ou outros fatores ambientais pudessem influenciar no rendimento das capturas.

Os autores utilizaram a armadilha New Jersey, contrariando seu emprego como instrumento de censo ou de monitoramento da fauna entomológica. Consideraram as coletas como um método para simular o efeito antrópico da iluminação artificial sobre os insetos. Duas armadilhas foram dotadas de lâmpadas com diferentes espectros, procurando-se testar o significado do espetro sobre a atração dos insetos.

Foram realizadas 11 coletas, durante o período de janeiro a abril de 2002 , sempre na ausência de lua no período da captura, para evitar que a claridade afetasse a atração dos insetos (Mazokhin-Purshnyakov, 1969) em duas distintas regiões: i) No Parque Ecológico do Tietê, município de Guarulhos, cerca de $14 \mathrm{Km}$ do centro da cidade de São Paulo. Foram realizadas sete capturas, utilizando-se duas armadilhas, instaladas em local arborizado, a $10 \mathrm{~m}$ uma da outra, próximas ao biotério, fora da influência de qualquer fonte de iluminação artificial. Durante as capturas foi possível verificar que a luminosidade do céu era muito elevada pela proximidade da cidade e do aeroporto internacional de Cumbica, entre os quais 
se encontra o parque. Este fato teria reduzido a capacidade de atração de longa distância exercida pelas armadilhas, porém, esse fator negativo seria compensado pela elevada densidade de culicídeos presente no local (Urbinatti et al., 2001; Taipe-Lagos, C.B. \& Natal, D. 2003). ii) No Interior paulista, a $160 \mathrm{Km}$ da capital, no município de Cordeirópolis, em uma chácara rural, a 500m do perímetro urbano, foram realizadas quatro capturas com armadilhas sendo colocadas a $10 \mathrm{~m}$ uma da outra, no centro de um gramado de $1000 \mathrm{~m}^{2}$, circundado de árvores frutíferas e cultura de milho. Não havia fontes de iluminação artificial e o ambiente mostrava-se pouco influenciado pelo reflexo luminoso da cidade.

As capturas foram realizadas sempre após o crepúsculo e estendiam-se por uma hora. Após cada coleta, os culicídeos eram mortos com clorofórmio, acondicionados em caixinhas entomológicas e transportados para o Laboratório de Entomologia da Faculdade de Saúde Pública/USP para a identificação. A captura com aspiração mecânica própria do equipamento utilizado, danificou alguns exemplares, o que prejudicou o reconhecimento taxonômico.

Os dados brutos foram convertidos em percentuais de indivíduos capturados, pela lâmpada fluorescente em relação à lâmpada incandescente. A transformação, retirando-se a aleatoriedade do número de capturas, intrínseco na própria distribuição dos insetos, reduziu a variabilidade da amostra, conferindo tratamento mais coerente dos dados.

\section{Resultados}

Em Cordeirópolis e no Parque Ecológico do Tietê, foram coletados respectivamente 150 e 656 espécimens de culicídeos (Tabs. I - III).

Nas tabelas I e III, referentes às coletas feitas em Cordeirópolis, em relação ao total de culicídeos coletados, observa-se tendência a um meIhor rendimento na armadilha com lâmpada fluorescente - 96 exemplares, 1,78 vezes em relação à lâmpada incandescente - 54 indivíduos. Se calcularmos a média pareada, esse valor aumenta para 1,99 vezes.

Devido ao baixo número de capturas, a comparação no nível de espécie não é estatisticamente significativa. Agrupando os indivíduos por gêneros, o rendimento na lâmpada fluorescente foi superior para Culex, Mansonia e Coquellettidia, nas duas localidades, enquanto para Anopheles foi superior apenas em Cordeirópolis. É significativo que em Cordeirópolis 
nas duas armadilhas o número de machos foi 7,3\% na armadilha fluorescente e de $18,5 \%$ na armadilha incandescente.

Em relação às coletas feitas no Parque Ecológico do Tietê, cujos resultados estão nas tabelas II e III, confirmou-se o maior rendimento associado à lâmpada fluorescente (366 espécimens), quando comparado à incandescente (290 espécimens), com um rendimento de 1,26 vezes na primeira, calculando o número absoluto, ou de 1,25 vezes quando se calcula a média pareada. O percentual de machos sobre o das fêmeas foi muito elevado, $30 \%$ na lâmpada fluorescente e $27 \%$ na incandescente.

\section{Tabela I}

Freqüência de culicídeos por espécies e sexos, segundo lâmpadas de diferentes naturezas, utilizadas em armadilhas New Jersey, em coletas feitas em Cordeirópolis, SP, de janeiro a abril de 2002.

\begin{tabular}{|c|c|c|c|c|c|c|c|}
\hline \multirow{2}{*}{ Espécies } & \multicolumn{3}{|c|}{ Lâmpada 9w } & \multicolumn{3}{|c|}{ Lâmpada 40w } & \multirow{2}{*}{ Total } \\
\hline & \& & $P$ & Total & o & P & Total & \\
\hline Aedeomyia (Aed.) squamipennis & 0 & 0 & 0 & 0 & 1 & 1 & 1 \\
\hline Aedes (Stg.) albopictus & 0 & 1 & 1 & 0 & 0 & 0 & 1 \\
\hline Ochlerototus (Och.) scapularis & 0 & 3 & 3 & 0 & 1 & 1 & 4 \\
\hline Anopheles (Nys.) albitarsis & 0 & 8 & 8 & 0 & 3 & 3 & 11 \\
\hline Anopheles (Nys.) strodei & 0 & 5 & 5 & 0 & 0 & 0 & 5 \\
\hline Anopheles (Nys.) parvus & 0 & 0 & 0 & 0 & 2 & 2 & 2 \\
\hline Anopheles (Nys.) evansae & 0 & 1 & 1 & 0 & 3 & 3 & 4 \\
\hline Anopheles sp. & 0 & 0 & 0 & 0 & 4 & 4 & 4 \\
\hline Culex (Cux.) sp. gr. Coronator & 4 & 21 & 25 & 0 & 6 & 6 & 31 \\
\hline Culex (Cux.) nigripalpus & 0 & 0 & 0 & 1 & 1 & 2 & 2 \\
\hline Culex (Cux.) chidesteri & 1 & 0 & 1 & 1 & 0 & 1 & 2 \\
\hline Culex (Cux.). prox.declarator & 0 & 0 & 0 & 4 & 1 & 5 & 5 \\
\hline Culex (Mel.) ocossa & 0 & 0 & 0 & 0 & 1 & 1 & 1 \\
\hline Culex (Mel.) ribeirensis & 1 & 3 & 4 & 0 & 1 & 1 & 5 \\
\hline Culex (Cux.) sp. & 1 & 3 & 4 & 4 & 0 & 4 & 8 \\
\hline Coquellettidia (Rhy.)venezuelensis & 0 & 5 & 5 & 0 & 1 & 1 & 6 \\
\hline Coquellettidia (Rhy.)juxtamansonia & 0 & 1 & 1 & 0 & 1 & 1 & 2 \\
\hline Coquellettidia sp. & 0 & 13 & 13 & 0 & 0 & 0 & 13 \\
\hline Uranotaenia (Ura.)lowi & 0 & 2 & 2 & 0 & 0 & 0 & 2 \\
\hline Anopheles sp.* & 0 & 20 & 20 & 0 & 14 & 14 & 34 \\
\hline Culex sp * & 0 & 3 & 3 & 0 & 0 & 0 & 3 \\
\hline Coquellettidia sp* & 0 & 0 & 0 & 0 & 4 & 4 & 4 \\
\hline Total & 7 & 89 & 96 & 10 & 44 & 54 & 150 \\
\hline$\% \varnothing \mathbf{e}$ & 7,3 & 92,7 & 100 & 18,5 & 81,5 & 100 & \\
\hline
\end{tabular}

* Danificados 


\section{Tabela II}

Freqüência de culicídeos por espécies e sexos, segundo lâmpadas de diferentes naturezas, utilizadas em armadilhas New Jersey, em coletas feitas no Parque Ecológico do Tietê, Guarulhos, SP, de janeiro a abril de 2002.

\begin{tabular}{|c|c|c|c|c|c|c|c|}
\hline \multirow{2}{*}{ Espécies } & \multicolumn{3}{|c|}{ Lâmpada 9w } & \multicolumn{3}{|c|}{ Lâmpada 40w } & \multirow{2}{*}{ Tota } \\
\hline & क & $Q$ & Total & d & $Q$ & Total & \\
\hline Aedes (Och.)serratus & 0 & 1 & 1 & 0 & 0 & 0 & 1 \\
\hline Ochlerototus (Och.)scapularis & 1 & 50 & 51 & 1 & 37 & 38 & 89 \\
\hline Anopheles (Nys.) albitarsis & 0 & 0 & 0 & 0 & 1 & 1 & 1 \\
\hline Anopheles (Nys.) strodei & 0 & 0 & 0 & 0 & 2 & 2 & 2 \\
\hline Anopheles (Nys.) evansae & 0 & 1 & 1 & 0 & 2 & 2 & 3 \\
\hline Anopheles (Nys.) sp. & 0 & 0 & 0 & 0 & 1 & 1 & 1 \\
\hline Culex (Cux.) gr. Coronator & 1 & 1 & 2 & 0 & 0 & 0 & 2 \\
\hline Culex (Cux.) nigripalpus & 83 & 57 & 140 & 5 & 1 & 6 & 146 \\
\hline Culex (Cux.) prox. declarator & 2 & 5 & 7 & 0 & 0 & 0 & 7 \\
\hline Culex (Cux.) sp. & 20 & 9 & 29 & 71 & 63 & 134 & 163 \\
\hline Mansonia (Man.) titillans & 3 & 31 & 34 & 0 & 7 & 7 & 41 \\
\hline Mansonia (Man.) prox. wilsoni & 0 & 0 & 0 & 0 & 1 & 1 & 1 \\
\hline Mansonia (Man.) sp. & 0 & 11 & 11 & 0 & 12 & 12 & 23 \\
\hline Coquellettidia (Rhy.)venezuelensis & 0 & 0 & 0 & 1 & 1 & 2 & 2 \\
\hline Uranotaenia (Ura.) lowi & 0 & 1 & 1 & 0 & 0 & 0 & 1 \\
\hline Uranotaenia (Ura.) geométrica & 0 & 1 & 1 & 0 & 0 & 0 & 1 \\
\hline Anopheles sp.* & 0 & 2 & 2 & 0 & 1 & 1 & 3 \\
\hline Coquellettidia sp.* & 0 & 2 & 2 & 0 & 0 & 0 & 2 \\
\hline Culex sp.* & 0 & 43 & 43 & 0 & 54 & 54 & 97 \\
\hline Mansonia sp. ${ }^{*}$ & 0 & 41 & 41 & 0 & 29 & 29 & 70 \\
\hline Total & 110 & 256 & 366 & 78 & 212 & 290 & 656 \\
\hline$\%$ e & 30 & 70 & 100 & 27 & 73 & 100 & \\
\hline
\end{tabular}

* Danificados

A figura 1 mostra a dispersão em um teste T por amostra pareada, no qual o percentual de indivíduos capturados na lâmpada fluorescente é em média $59,2 \%$ do total, contra 40,8 \% da lâmpada incandescente.

\section{Discussão}

Os resultados dos dois sítios de captura revelam evidente diferença no rendimento da lâmpada fluorescente em relação à incandescente: 462 


\section{Tabela III}

Culicídeos capturados com armadilhas New Jersey, segundo tipos de

lâmpadas nas diferentes coletas realizadas nos municípios de

Cordeirópolis, SP e Parque Ecológico do Tietê, Guarulhos, SP, no período de janeiro a abril de 2002.

\begin{tabular}{|c|c|c|c|c|c|}
\hline \multirow{7}{*}{$\begin{array}{l}\frac{0}{\overline{0}} \\
\frac{0}{0} \\
\frac{\overline{0}}{\overline{0}} \\
\frac{0}{0} \\
0\end{array}$} & \multirow{2}{*}{ Data } & \multirow{2}{*}{ Col. N ${ }^{\circ}$. } & \multicolumn{2}{|c|}{ Tipos de lâmpadas } & \multirow[b]{2}{*}{ Fluor/Inc } \\
\hline & & & Fluorescente & Incandescente & \\
\hline & $04-02-02$ & $3-4$ & 14 & 5 & 2,80 \\
\hline & $22-02-02$ & $5-6$ & 25 & 12 & 2,08 \\
\hline & $23-02-02$ & $7-8$ & 32 & 19 & 1,68 \\
\hline & $25-03-02$ & $9-10$ & 25 & 18 & 1,39 \\
\hline & Sub Total & & 96 & 54 & 1,78 \\
\hline \multirow{12}{*}{ 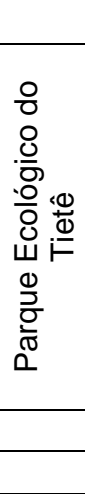 } & Média pareada & & & & 1,99 \\
\hline & $28 / 01 / 02$ & 01-02 & 16 & 13 & 1,23 \\
\hline & $01 / 04 / 02$ & $11-12$ & 47 & 38 & 1,24 \\
\hline & 02/04/02 & $13-14$ & 38 & 35 & 1,09 \\
\hline & 04/04/02 & $15-16$ & 48 & 39 & 1,23 \\
\hline & 05/04/02 & $17-18$ & 67 & 62 & 1,08 \\
\hline & $11 / 04 / 02$ & $19-20$ & 78 & 54 & 1,44 \\
\hline & $12 / 04 / 02$ & $21-22$ & 72 & 49 & 1,47 \\
\hline & Sub Total & & 366 & 290 & 1,26 \\
\hline & Média pareada & & & & 1,25 \\
\hline & Total & & 462 & 344 & 1,34 \\
\hline & Média pareada & & & & 1,52 \\
\hline
\end{tabular}

indivíduos contra 344, isto é, 1,34 vezes mais indivíduos. Calculando-se a média dos dados pareados, esse valor aumenta para 1,52 vezes.

Os dados da captura de Guarulhos são sem dúvida menos significativos que os de Cordeirópolis e merecem comentário. A pequena diferença de indivíduos capturados entre as duas lâmpadas pode ter duas explicações. A primeira é que a claridade da abóbada celeste reduz o efeito da atração e portanto o elevado número de mosquitos capturados é apenas a conseqüência da alta concentração dos mesmos. A segunda é que o ambiente arborizado no qual foram realizadas as capturas, não permitiu aos mosquitos orientar seu vôo a uma ou outra lâmpada. Por essa razão esses dípteros se dirigiam com mais freqüência para a lâmpada mais próxima, diminuindo a seletividade da atração.

Os resultados, apesar de parciais, indicaram diferença de atratividade ao se utilizar lâmpadas fluorescentes e incandescentes, sendo as primei- 


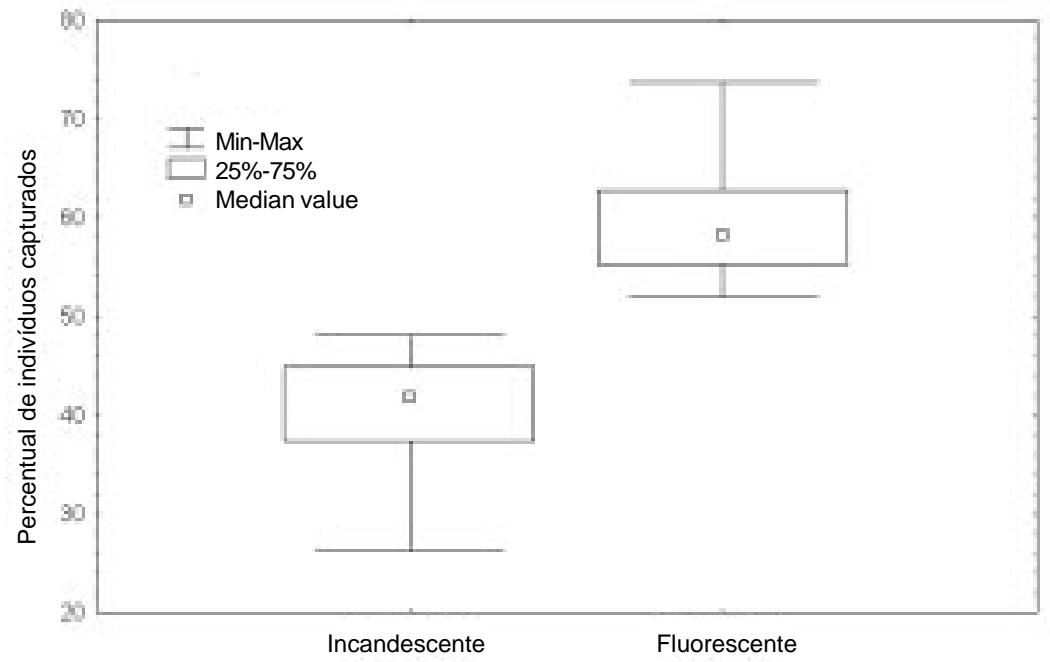

Figura 1: Teste por amostras dependentes do percentual de indivíduos capturados, segundo lâmpadas de diferentes naturezas, utilizadas em armadilhas New Jersey, em coletas feitas em Cordeirópolis, SP e Parque Ecológico do Tietê, Guarulhos, SP, no período de janeiro a abril de 2002.

ras as que atraem com mais eficiência os culicídeos, como previsto pelos conhecimentos atuais da fisiologia da visão dos insetos. A única referência encontrada na literatura sobre os efeitos da iluminação na transmissão de patógenos relata uma infecção por Tripanosoma cruzi contraída em Belém do Pará, que teria sido veiculada por triatomíneo atraído pela luz (Miles et al., 1981)

Para se obter resultados mais consistentes, seria necessário um investimento maior nas capturas, o que não foi possível dada às limitações de recursos para essa pesquisa. As lâmpadas comerciais são de elevada diversidade e, portanto, seriam necessários testes preliminares para definição dos espectros daquelas mais utilizadas. Atendido esse requisito, poder-se-á viabilizar projetos mais aprofundados para estudos das diferentes respostas dos culicídeos em investigações que cubram os ciclos: sazonais, lunares e nictimerais. Para um programa dessa envergadura, seria necessária a cooperação de diferentes segmentos da sociedade ao serem acampados os setores: elétrico, de saúde e do ambiente. 
A divulgação desses dados iniciais, apesar de limitados, oferece uma primeira demonstração dos possíveis impactos que a iluminação artificial deva estar exercendo em áreas remotas e servirá de estímulo para a conquista de desafios maiores.

\section{Conclusão}

A atração de culicídeos em teste de campo revelou que as lâmpadas fluorescentes foram significativamente mais atrativas que as incandescentes.

A iluminação artificial deve ser considerada mais um elemento de impacto antrópico que merece ser estudado antes da execução de programas de eletrificação.

Há necessidade de ampliar os estudos comparativos de lâmpadas de diferentes aspectos, quanto à atração de culicídeos.

\section{Agradecimentos}

Ao Prof. Dr. Sergio Vanin, IB, USP, e à Prof ${ }^{a}$. Dr. Miriam D. Marques, do Museu de Zoologia de S.P, pelo apoio e pelas preciosas sugestões, ao Eng. Isac Rosemblat, pelas discussões sobre as características das lâmpadas e à física Jane Cleide Goveia, do Instituto de Pesquisas Tecnológicas do IPT, que realizou as medições de irradiância das lâmpadas. Especial agradecimento à Diretora Herle da Costa Bezerra, e funcionários do Parque Ecológico do Tietê, São Paulo, por ter permitido a execução dos testes e pelo apoio logístico.

\section{Referências Bibliográficas:}

Bergman, R.S. Jr.; Parham, T.G \& McGowan, U.V., 1995. UV emission from general lighting Lamps, J. Illum. Soc. 24:13-24.

Brioscoe, A.D. \& Chittka, L., 2001. The evolution of color vision in insects. Ann. Rev. Entomol. 46:471-510. 
Clements, A.N., 1999. The biology of mosquitoes: Sensory reception and behaviour. 2. Wallingford; Caby, London. $740 \mathrm{p}$.

Dethier, V.G., 1963. The physiology of insects senses. Wiley, London. $266 \mathrm{p}$.

Goldsmith, T.H. 1970 The visual system of insects, in Rockstein, Physiol. Ins. 2:165-272.

Gould J.L. \& Gould C.G.,1988. The honey bee. Scientific American Labrary, New York. 239 p.

IESNA, 1993. Lighting handbook, reference and applications (Rea Marks eds), $8^{\text {th }}$ ed. Illuminating Engineering Society of North America, New York. 989 p.

Mazokhin-Porshnyakov, G.A., 1969. Insect vision. Plenum Press, New York. $306 \mathrm{p}$.

McGowan, T., 1989. Energy efficient lighting in electricity p. 59-88. In: Eficient end-use and new generation technologies and their planning implications (T. Joahansson, B. Boudlund e R. Williams, eds.) Lund University Press, Lund. $960 \mathrm{p}$.

Miles, M.A., Souza, A.A. \& Póvoa M., 1981. Chagas disease in the amazon basin III. Ecotopes of ten triatomine bug species (Hemiptera: Reduviidae) from the vicinity of Belém, Pará State, Brazil. J. Med. Entomol. 18:266-278.

Reinert, W.C., 1989. The New Jersey Light Trap: An old standard for most mosquito control programs. Proceedings of the Seventy-Sixth Annual Meeting of the New Jersey Mosquito Control Association [on line] p.17-25. Disponível em <http://www.rci.rutgers.edu/ insects/njtrap.htm/>.

Service, M.W.,1993. Mosquito ecology field sampling methods. 2nd edition, Chapman \& Hall, London. 989 p. 
Taipe-Lagos, C.B. \& Natal, D., 2003. Abundância de culicídeos em área metropolitana preservada e suas implicações epidemiológicas. Rev. Saude Publica. 37:275-279.

Urbinatti, P.R.; Sendacz,S. \& Natal, D.,2001. Imaturos de mosquitos (Diptera: Culicidae) em parque de área metropolitana aberto à visitação pública. Rev. Saude Publica. 35:461-466. 